\title{
Atomic data from the Iron Project. XVI. Photoionization cross sections and oscillator strengths for Fe V
}

\author{
M.A. Bautista \\ Department of Astronomy, The Ohio State University, 174 West 18th Avenue, Columbus, OH 43210-1106, U.S.A. \\ Internet: bautista@payne.mps.ohio-state.edu
}

Received December 19, 1995; accepted February 15, 1996

\begin{abstract}
Large scale ab initio calculations of photoionization and dipole transition probabilities for Fe $\mathrm{V}$ are reported. The computations were carried out in the close coupling approximation using the R-matrix method including $34 \mathrm{LS}$ terms of the target ion Fe VI. All bound states of Fe V with $n \leq 10$ and $l \leq 7$ are considered. The results include 1816 LS bound states, dipole oscillator strengths for 129.904 LS multiplets, and detailed photoionization cross sections with extensive resonance structures for all of the bound states. These data differ significantly in many cases from the earlier data calculated under the Opacity Project and other theoretical calculations. It is expected that the new data should yield more accurate Iron opacities, particularly in objects where Fe V is observed to be one of the dominant species, such as the atmospheres of hot, young white dwarfs at temperatures of $40.000-60.000 \mathrm{~K}$. The present calculations were mainly carried out on the massively parallel Cray T3D with a parallelized version of the Iron Project R-matrix codes.
\end{abstract}

Key words: atomic data — plasmas — ultraviolet general — infrared general

\section{Introduction}

Low ionization stages of iron, owing to their rich and complex structure and their relatively high abundance, dominate certain wavelength regions in the spectra of a variety of stellar and non-stellar objects, as well as the interstellar medium. For example, Fe $\mathrm{V}$ is one of the most important ions in the EUV spectra of emitting photospheres of hot, young white dwarfs, as recently observed by the Extreme Ultraviolet Explorer (Pradhan 1995; Vennes 1995). The interpretation and modeling of these observations rely almost entirely on theoretical values of the atomic data, which until the recent advances made under the Opacity Project (OP, Seaton et al. 1994) and the Iron Project (IP, Hummer et al. 1993) could not be obtained either with sufficient accuracy or on the large scale needed for a full determination of the relevant astrophysical parameters. However, some of the Opacity Project calculations, particularly for the low ionization stages of Iron including $\mathrm{Fe} \mathrm{V}$, are of insufficient accuracy and consequently lead to significant discrepancies between models and observations (e.g. Pradhan 1995). It is one of the aims of the Iron Project to carry out improved calculations for these ions (Bautista et al. 1995) and results for Fe II have already been reported (e.g. Nahar \& Pradhan 1994;
Nahar 1995a); and, for Fe I and Fe III the calculations have been recently completed and are being reported separately (Bautista 1996; Nahar 1996).

Atomic calculations for these low ionization stages of Iron, i.e. Fe I - Fe VI, are difficult due to the complexity of the electron-electron correlation effects, coupling effects, associated resonance structures, and the large number of states involved. The computational requirements are very intensive; however, the advent of massively parallel processors (MPP's) is an important development that promises to substantially address these needs. In a previous work, we presented the first ab-initio calculations using the Rmatrix package of codes (Berrington et al. 1987) on the MPP Cray T3D for electron impact excitation of Ni II and Fe II (Bautista \& Pradhan 1996). In the present work we report a large scale computation of radiative data for $\mathrm{Fe} \mathrm{V}$, also carried out on the Cray T3D.

\section{Target expansion}

In the close coupling (CC) approximation the total wave function of the (e+ion) system is represented as

$$
\Psi(E ; S L \pi)=A \sum_{i} \chi_{i} \theta_{i}+\sum_{j} c_{j} \Phi_{j},
$$


Table 1. Calculated (cal) energy levels of Fe VI and comparison with observed (obs) levels. The energies (in Rydberg) are relative to the $3 \mathrm{~d}^{3}\left({ }^{3} \mathrm{~F}\right)$ ground state. The spectroscopic and correlation configurations for Fe VI, and the values of the scaling parameters $\lambda_{n l}$ for each orbital in the Thomas-Fermi-Dirac potential used in Superstructure are also given

\begin{tabular}{|c|c|c|c|c|c|c|c|c|c|}
\hline & Level & & $E_{\text {obs }}$ & $E_{\text {cal }}$ & & Level & & $E_{\text {obs }}$ & $E_{\text {cal }}$ \\
\hline 1 & $3 d^{3}$ & ${ }^{4} \mathrm{~F}$ & 0.000000 & 0.000000 & 18 & $3 \mathrm{~d}^{2}\left({ }^{3} \mathrm{~F}\right) 4 \mathrm{p}$ & ${ }^{2} \mathrm{~F}^{0}$ & 3.11654 & 3.157376 \\
\hline 2 & $3 d^{3}$ & ${ }^{4} \mathrm{P}$ & 0.164747 & 0.169518 & 19 & $3 d^{2}\left({ }^{3} F\right) 4 p$ & ${ }^{4} \mathrm{D}^{0}$ & 3.12830 & 3.163145 \\
\hline 3 & $3 d^{3}$ & ${ }^{2} \mathrm{G}$ & 0.180803 & 0.193499 & 20 & $3 d^{2}\left({ }^{3} F\right) 4 p$ & ${ }^{2} \mathrm{D}^{0}$ & 3.13696 & 3.168952 \\
\hline 4 & $3 d^{3}$ & ${ }^{2} \mathrm{P}$ & 0.229137 & 0.232475 & 21 & $3 d^{2}\left({ }^{3} F\right) 4 p$ & ${ }^{2} \mathrm{G}^{0}$ & 3.17472 & 3.219006 \\
\hline 5 & $3 d^{3}$ & ${ }^{2} \mathrm{D}$ & 0.249488 & 0.242133 & 22 & $3 \mathrm{~d}^{2}\left({ }^{3} \mathrm{P}\right) 4 \mathrm{p}$ & ${ }^{2} \mathrm{~S}^{0}$ & 3.19529 & 3.241274 \\
\hline 6 & $3 d^{3}$ & ${ }^{2} \mathrm{H}$ & 0.253531 & 0.281387 & 23 & $3 d^{2}\left({ }^{3} \mathrm{P}\right) 4 \mathrm{p}$ & ${ }^{4} \mathrm{~S}^{0}$ & 3.23038 & 3.283241 \\
\hline 7 & $3 d^{3}$ & ${ }^{2} \mathrm{~F}$ & 0.412069 & 0.435277 & 24 & $3 d^{2}\left({ }^{1} \mathrm{D}\right) 4 \mathrm{p}$ & ${ }^{2} \mathrm{P}^{0}$ & 3.25359 & 3.299941 \\
\hline 8 & $3 d^{3}$ & ${ }^{2} \mathrm{D}$ & 0.644089 & 0.655111 & 25 & $3 \mathrm{~d}^{2}\left({ }^{1} \mathrm{D}\right) 4 \mathrm{p}$ & ${ }^{2} \mathrm{~F}^{0}$ & 3.26285 & 3.314892 \\
\hline 9 & $3 d^{2}\left({ }^{3} F\right) 4 s$ & ${ }^{4} \mathrm{~F}$ & 2.38728 & 2.328184 & 26 & $3 d^{2}\left({ }^{3} \mathrm{P}\right) 4 \mathrm{p}$ & ${ }^{4} \mathrm{D}^{0}$ & 3.27922 & 3.322854 \\
\hline 10 & $3 \mathrm{~d}^{2}\left({ }^{3} \mathrm{~F}\right) 4 \mathrm{~s}$ & ${ }^{2} \mathrm{~F}$ & 2.44996 & 2.414726 & 27 & $3 \mathrm{~d}^{2}\left({ }^{1} \mathrm{D}\right) 4 \mathrm{p}$ & ${ }^{2} \mathrm{D}^{0}$ & 3.29096 & 3.346683 \\
\hline 11 & $3 d^{2}\left({ }^{1} D\right) 4 s$ & ${ }^{2} \mathrm{D}$ & 2.55031 & 2.516728 & 28 & $3 \mathrm{~d}^{2}\left({ }^{3} \mathrm{P}\right) 4 \mathrm{p}$ & ${ }^{4} \mathrm{P}^{0}$ & 3.31433 & 3.362279 \\
\hline 12 & $3 d^{2}\left({ }^{3} P\right) 4 s$ & ${ }^{4} \mathrm{P}$ & 2.56282 & 2.518222 & 29 & $3 \mathrm{~d}^{2}\left({ }^{1} \mathrm{G}\right) 4 \mathrm{p}$ & ${ }^{2} \mathrm{G}^{0}$ & 3.31718 & 3.356075 \\
\hline 13 & $3 d^{2}\left({ }^{3} \mathrm{P}\right) 4 \mathrm{~s}$ & ${ }^{2} \mathrm{P}$ & 2.61748 & 2.600714 & 30 & $3 d^{2}\left({ }^{3} \mathrm{P}\right) 4 \mathrm{p}$ & ${ }^{2} \mathrm{D}^{0}$ & 3.36622 & 3.442309 \\
\hline 14 & $3 d^{2}\left({ }^{1} G\right) 4 s$ & ${ }^{2} \mathrm{G}$ & 2.65322 & 2.604507 & 31 & $3 \mathrm{~d}^{2}\left({ }^{1} \mathrm{G}\right) 4 \mathrm{p}$ & ${ }^{2} \mathrm{H}^{0}$ & 3.38819 & 3.421722 \\
\hline 15 & $3 d^{2}\left({ }^{3} F\right) 4 p$ & ${ }^{2} \mathrm{~S}$ & 3.06587 & 2.95662 & 32 & $3 \mathrm{~d}^{2}\left({ }^{3} \mathrm{P}\right) 4 \mathrm{p}$ & ${ }^{2} \mathrm{P}^{0}$ & 3.40039 & 3.477487 \\
\hline 16 & $3 d^{2}\left({ }^{3} F\right) 4 p$ & ${ }^{4} \mathrm{G}^{0}$ & 3.09326 & 3.117651 & 33 & $3 \mathrm{~d}^{2}\left({ }^{1} \mathrm{G}\right) 4 \mathrm{p}$ & ${ }^{2} \mathrm{~F}^{0}$ & 3.43794 & 3.487353 \\
\hline 17 & $3 \mathrm{~d}^{2}\left({ }^{3} \mathrm{~F}\right) 4 \mathrm{p}$ & ${ }^{4} \mathrm{~F}^{0}$ & 3.09926 & 3.128948 & 34 & $3 \mathrm{~d}^{2}\left({ }^{1} \mathrm{~S}\right) 4 \mathrm{p}$ & ${ }^{2} \mathrm{P}^{0}$ & 3.72251 & 3.788938 \\
\hline
\end{tabular}

Fe VI configurations

Spectroscopic: $3 \mathrm{~s}^{2} 3 \mathrm{p}^{6} 3 \mathrm{~d}^{3}, 3 \mathrm{~s}^{2} 3 \mathrm{p}^{6} 3 \mathrm{~d}^{2} 4 \mathrm{~s}, 3 \mathrm{~s}^{2} 3 \mathrm{p}^{6} 3 \mathrm{~d}^{2} 4 \mathrm{p}$

Correlation: $3 \mathrm{~s}^{2} 3 \mathrm{p}^{5} 3 \mathrm{~d}^{4}, 3 \mathrm{~s}^{2} 3 \mathrm{p}^{4} 3 \mathrm{~d}^{5}, 3 \mathrm{~s} 3 \mathrm{p}^{6} 3 \mathrm{~d}^{4}, 3 \mathrm{p}^{6} 3 \mathrm{~d}^{5}, 3 \mathrm{p}^{6} 3 \mathrm{~d}^{4} 4 \mathrm{~s}, 3 \mathrm{~s}^{2} 3 \mathrm{p}^{6} 3 \mathrm{~d}^{2} 4 \mathrm{~d}$

$\lambda_{n l}$ : 1.1024 (1s), 1.0867 (2s), 1.4669 (2p), 1.4669 (3s), 1.1000 (3p), 1.0640 (3d), 1.1655 (4s), 1.0968 (4p), 1.2287 (4d).

where $\chi_{i}$ is the target ion wave function in a specific state $S_{i} L_{i}, \theta_{i}$ is the wave function for the free electron, and $\Phi_{j}$ are short range correlation functions for the bound (e+ion) system. Accurate CC calculations of atomic processes require, first of all, a good representation of the core ion. Such representation of complex ions has to include a large number of correlation configurations. However, in order for the computations to be computationally tractable, the configuration expansion needs to be as small as practical.

The CC expansion for the present calculation includes $34 \mathrm{LS}$ terms of the target or core ion Fe VI.

The list of all terms included for this calculation is given in Table 1.

The atomic structure code SUPERSTRUCTURE (Eissner et al. 1974; Eissner 1991) was used in the present work to compute eigenfunctions of the 34 states of the Fe VI target ion dominated by the configurations $3 \mathrm{~d}^{3}$, $3 \mathrm{~d}^{2} 4 \mathrm{~s}$, and $3 \mathrm{~d}^{2} 4 \mathrm{p}$. Table 1 presents the complete list of configurations included in the target, as well as a comparison between the calculated target term energies and the observed energies, averaged over the fine structure, taken from Sugar \& Corliss (1985). The agreement between the energies is very good, generally within $2 \%$. Another indicator of the accuracy of the target representation is the good agreement between the length and the velocity $f$ values, which for the present case is typically about $5 \%$.

\section{Radiative calculations}

The present calculations were carried out in the LS coupling scheme. Overall we expect the relativistic effects in the low ionization stages of iron to be small. Although they might be significant for some types of transitions in $\mathrm{Fe} \mathrm{V}$, it would be impractical at this stage to carry through a large-scale relativistic calculation involving several times the number of channels than the already huge number in the LS coupling case. Also, the inclusion of fine structure has a marginal effect on the calculation of the Rosseland or the Planck mean opacities (Seaton et al. 1994).

Table 2. Correlation functions for $\mathrm{Fe} \mathrm{V}$ included in the $\mathrm{CC}$ expansion

$3 \mathrm{~s}^{2} 3 \mathrm{p}^{6} 3 \mathrm{~d}^{4}, 3 \mathrm{~s}^{2} 3 \mathrm{p}^{6} 3 \mathrm{~d}^{3} 4 \mathrm{~s}, 3 \mathrm{~s}^{2} 3 \mathrm{p}^{6} 3 \mathrm{~d}^{3} 4 \mathrm{p}, 3 \mathrm{~s}^{2} 3 \mathrm{p}^{6} 3 \mathrm{~d}^{3} 4 \mathrm{~d}, 3 \mathrm{~s}^{2} 3 \mathrm{p}^{5} 3 \mathrm{~d}^{5}$, $3 \mathrm{~s}^{2} 3 \mathrm{p}^{5} 3 \mathrm{~d}^{4} 4 \mathrm{~s}, 3 \mathrm{~s}^{2} 3 \mathrm{p}^{5} 3 \mathrm{~d}^{4} 4 \mathrm{~d}, 3 \mathrm{~s}^{2} 3 \mathrm{p}^{5} 3 \mathrm{~d}^{3} 4 \mathrm{~s} 4 \mathrm{~d}, 3 \mathrm{p}^{5} 3 \mathrm{~d}^{5} 4 \mathrm{~s} 4 \mathrm{~d}$, $3 \mathrm{p}^{6} 3 \mathrm{~d}^{6}, 3 \mathrm{p}^{6} 3 \mathrm{~d}^{5} 4 \mathrm{p}, 3 \mathrm{~s}^{2} 3 \mathrm{p}^{4} 3 \mathrm{~d}^{6}, 3 \mathrm{~s}^{2} 3 \mathrm{p}^{4} 3 \mathrm{~d}^{5} 4 \mathrm{p}, 3 \mathrm{~s} 3 \mathrm{p}^{6} 3 \mathrm{~d}^{3} 4 \mathrm{~s} 4 \mathrm{~d}$, $3 \mathrm{p}^{6} 3 \mathrm{~d}^{3} 4 \mathrm{~s}^{2} 4 \mathrm{~d}, 3 \mathrm{p}^{6} 3 \mathrm{~d}^{3} 4 \mathrm{~s} 4 \mathrm{p}^{2}, 3 \mathrm{~s}^{2} 3 \mathrm{p}^{4} 3 \mathrm{~d}^{4} 4 \mathrm{p}^{2}, 3 \mathrm{~s}^{2} 3 \mathrm{p}^{4} 3 \mathrm{~d}^{4} 4 \mathrm{~s}^{2}$, $3 \mathrm{~s} 3 \mathrm{p}^{6} 3 \mathrm{~d}^{4} 4 \mathrm{~s}, 3 \mathrm{~s} 3 \mathrm{p}^{6} 3 \mathrm{~d}^{4} 4 \mathrm{~d}$

The second summation in the CC expansion (Eq. 1) represents short range correlation functions. These functions are very important in obtaining accurate $(\mathrm{e}+$ ion $)$ wavefunctions, but may result in pseudoresonances, particularly if the two summations in Eq. (1) are inconsistent 
(Berrington et al. 1987). In the present work it was necessary to optimize the set of $\Phi_{j}$ functions for $\mathrm{Fe} \mathrm{V}$ in such a way that only a minimum number of correlation functions with important effects on the bound states energies and bound-bound oscillator strengths for Fe $\mathrm{V}$ was included. A list of all correlation functions included in the CC expansion is given in Table 2 .

The overall calculation was divided into groups of total (e + ion) symmetries SL $\pi$ according to their multiplicity, i.e. $(2 \mathrm{~S}+1)=1,3$, and 5 . For each set of $(2 \mathrm{~S}+1)$ multiplicity we consider total angular momenta $L=0-7$, for both parities, and a total of 34 SL $\pi$ 's corresponding to the target states in Table 1.

\section{Results}

Three sets of data are calculated: (a) energy levels, (b) oscillator strengths, and (c) total and partial photoionization cross sections.

\subsection{Energy levels}

As a first step energies for bound state terms of $\mathrm{Fe} \mathrm{V}$ were obtained. A total of 1818 states in LS coupling were found up to $n \leq 10$. In Table 3 we compare the computed energies for these terms with the few experimental values available (Sugar \& Corliss 1985). The overall agreement is of about $1.6 \%$; the difference for the $3 \mathrm{~d}^{4}\left({ }^{5} \mathrm{D}\right)$ ground state is only of $0.85 \%$. In the same table we also present the unpublished results computed by Butler under the OP in TOPbase at CDS (Cunto et al. 1993) using a 15 CC expansion. These values differe from the observed energies by about $2.5 \%$ which is sligthly worst than for the present results.

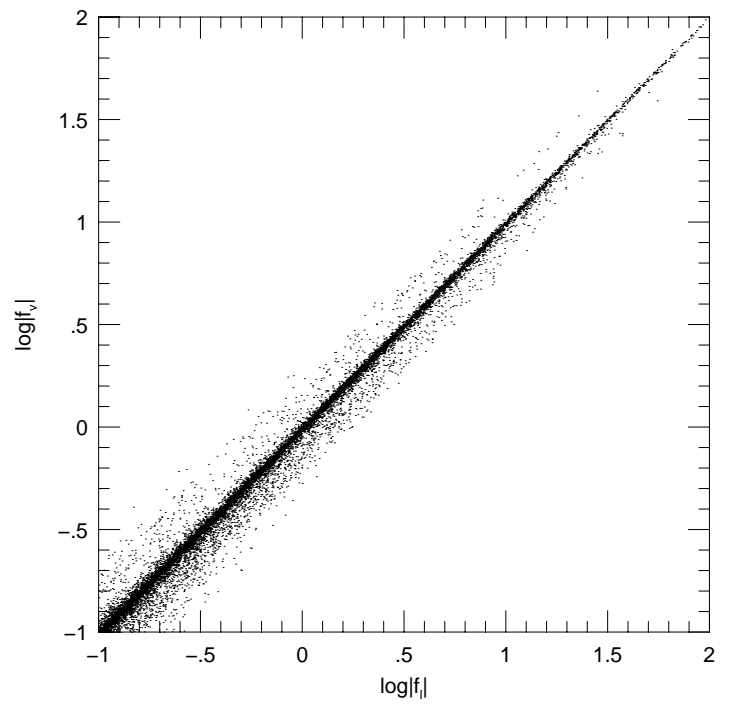

Fig. 1. $\log g f_{V}$ plotted against $\log g f_{L}$ for transitions between calculated LS terms
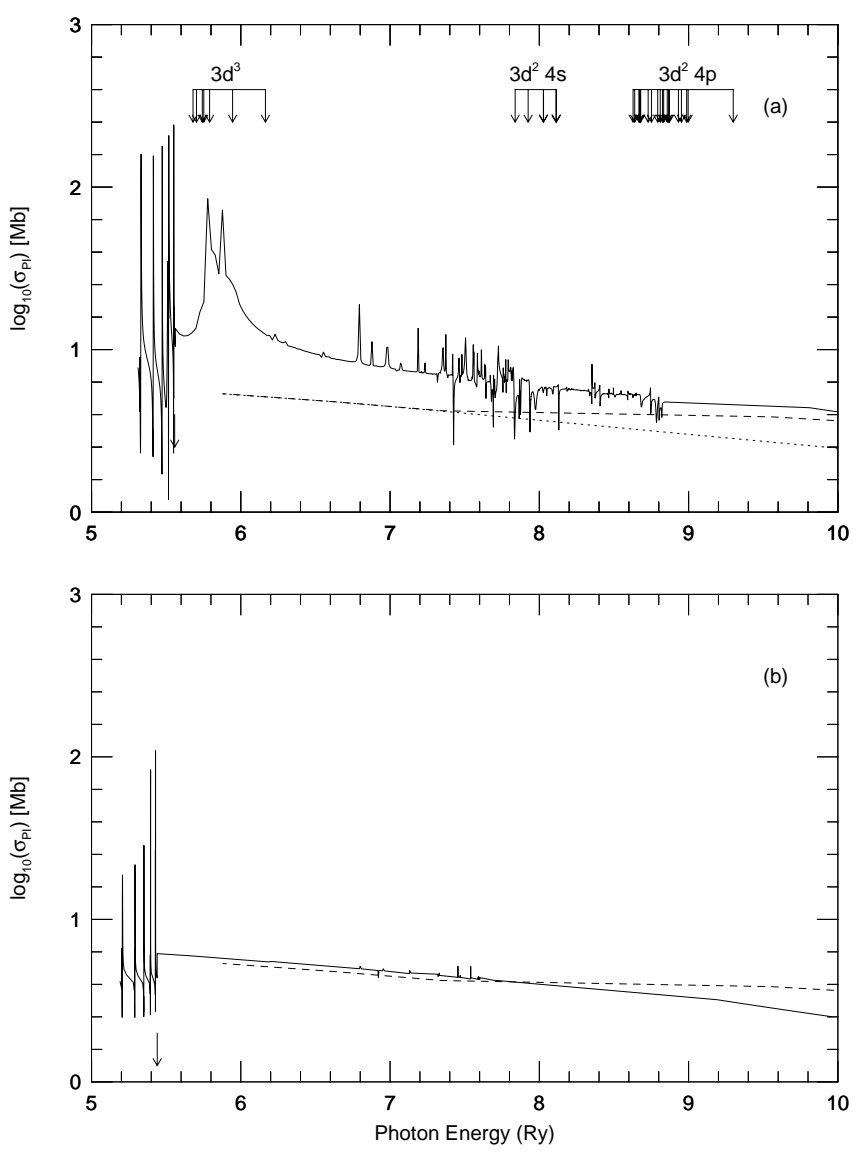

Fig. 2. Photoionization cross section $(\sigma(\mathrm{Mb}))$ as a function of photon energy in Ry of th ground state $3 \mathrm{p}^{4}\left({ }^{5} \mathrm{D}\right)$ of Fe V. Panel a) compares the present results (solid line) with the results by Reilman \& Manson (1979; dashed line) and Verner et al. (1993; dot-dashed line). In panel b) cross sections by Butler in TOPbase (Cunto et al. 1993, solid line) and by Reilman \& Manson (1979; dashed line)

\subsection{Oscillator strengths}

Dipole oscillator strengths ( $f$-values) for 129.904 transitions among the calculated states of $\mathrm{Fe} \mathrm{V}$ were obtained in LS coupling. This set includes transitions for which the lower state lies below the first ionization threshold and the upper state lies above. These transitions are important because they might contribute to the total photo-absorption, but do not appear as resonances in the photoionization cross sections.

In the absence of experimentally measured $f$-values it is difficult to ascertain the overall accuracy of the data. However, a comparison of length and velocity oscillator strengths provides us with a check on the accuracy of the wavefunctions and, therefore, on the reliability of the $f$ values. In Fig. 1 we plot $\log g f_{V}$ vs. $\log g f_{L}$. In this plot we have included all the symmetries since each of them exhibits roughly the same dispersion. The agreement between length and velocity $f$-values is quite satisfactory, 
Table 3. Comparison of calculated (cal) energies in Ry with the results by Butler in TOPbase at CDS (Cunto et al. 1993; TOP) an the observed (obs) energies from Sugar \& Corliss (1985)

\begin{tabular}{|c|c|c|c|c|c|c|c|c|c|}
\hline Conf. & Term & $E_{\text {cal }}$ & $E_{\mathrm{TOP}}$ & $E_{\text {obs }}$ & Conf. & Term & $E_{\text {cal }}$ & $E_{\mathrm{TOP}}$ & $E_{\text {obs }}$ \\
\hline $3 \mathrm{~d}^{4}$ & ${ }^{5} \mathrm{D}$ & -5.557 & -5.440 & -5.510 & $3 \mathrm{~d}^{3}\left({ }^{4} \mathrm{P}\right) 4 \mathrm{~s}$ & ${ }^{3} \mathrm{P}$ & -3.495 & -3.484 & -3.574 \\
\hline $3 d^{4}$ & ${ }^{3} \mathrm{P}$ & -5.342 & -5.183 & -5.283 & $3 d^{3}\left({ }^{2} G\right) 4 s$ & ${ }^{1} \mathrm{G}$ & -3.503 & -3.495 & -3.571 \\
\hline $3 d^{4}$ & ${ }^{3} \mathrm{H}$ & -5.318 & -5.212 & -5.287 & $3 d^{3}\left({ }^{2} \mathrm{P}\right) 4 \mathrm{~s}$ & ${ }^{3} \mathrm{P}$ & -3.482 & -3.472 & -3.562 \\
\hline $3 d^{4}$ & ${ }^{3} \mathrm{~F}$ & -5.314 & -5.176 & -5.272 & $3 d^{3}\left({ }^{2} D\right) 4 s$ & ${ }^{3} \mathrm{D}$ & -3.467 & -3.446 & -3.545 \\
\hline $3 d^{4}$ & ${ }^{3} \mathrm{G}$ & -5.272 & -5.151 & -5.242 & $3 d^{3}\left({ }^{2} \mathrm{H}\right) 4 \mathrm{~s}$ & ${ }^{3} \mathrm{H}$ & -3.471 & -3.470 & -3.540 \\
\hline $3 d^{4}$ & ${ }^{1} \mathrm{G}$ & -5.217 & -5.074 & -5.184 & $3 d^{3}\left({ }^{2} \mathrm{P}\right) 4 \mathrm{~s}$ & ${ }^{1} \mathrm{P}$ & -3.446 & -3.434 & -3.517 \\
\hline $3 d^{4}$ & ${ }^{3} \mathrm{D}$ & -5.203 & -5.064 & -5.182 & $3 d^{3}\left({ }^{2} \mathrm{D} 2\right) 4 \mathrm{~s}$ & ${ }^{1} \mathrm{D}$ & -3.429 & -3.406 & -3.507 \\
\hline $3 d^{4}$ & ${ }^{1} \mathrm{I}$ & -5.197 & & -5.175 & $3 \mathrm{~d}^{3}\left({ }^{2} \mathrm{H}\right) 4 \mathrm{~s}$ & ${ }^{1} \mathrm{H}$ & -3.431 & -3.430 & -3.500 \\
\hline $3 d^{4}$ & ${ }^{1} \mathrm{~S}$ & -5.188 & -5.009 & -5.156 & $3 d^{3}\left({ }^{2} F\right) 4 s$ & ${ }^{3} \mathrm{~F}$ & -3.302 & -3.280 & -3.387 \\
\hline $3 d^{4}$ & ${ }^{1} \mathrm{D}$ & -5.149 & -4.988 & -5.095 & $3 d^{3}\left({ }^{2} F\right) 4 s$ & ${ }^{1} \mathrm{~F}$ & -3.268 & -3.243 & -3.351 \\
\hline $3 d^{4}$ & ${ }^{1} \mathrm{~F}$ & -5.059 & -4.915 & -5.037 & $3 d^{3}\left({ }^{4} F\right) 4 p$ & ${ }^{5} \mathrm{G}^{0}$ & -3.181 & -3.135 & -3.178 \\
\hline $3 d^{4}$ & ${ }^{3} \mathrm{P}$ & -4.980 & -4.814 & -4.949 & $3 d^{3}\left({ }^{4} F\right) 4 p$ & ${ }^{5} \mathrm{D}^{0}$ & -3.152 & -3.110 & -3.159 \\
\hline $3 d^{4}$ & ${ }^{3} \mathrm{~F}$ & -4.982 & -4.823 & -4.949 & $3 d^{3}\left({ }^{2} \mathrm{D} 1\right) 4 \mathrm{~s}$ & ${ }^{3} \mathrm{D}$ & -3.038 & -3.021 & -3.161 \\
\hline $3 d^{4}$ & ${ }^{1} \mathrm{G}$ & -4.895 & -4.741 & -4.868 & $3 d^{3}\left({ }^{4} F\right) 4 p$ & ${ }^{5} \mathrm{~F}^{0}$ & -3.142 & -3.099 & -3.144 \\
\hline $3 d^{4}$ & ${ }^{1} \mathrm{D}$ & -4.673 & -4.494 & -4.662 & $3 d^{3}\left({ }^{4} \mathrm{~F}\right) 4 \mathrm{p}$ & ${ }^{3} \mathrm{D}^{0}$ & -3.142 & -3.102 & -3.142 \\
\hline $3 d^{4}$ & ${ }^{1} \mathrm{~S}$ & -4.446 & -4.215 & -4.413 & $3 d^{3}\left({ }^{2} \mathrm{D} 1\right) 4 \mathrm{~s}$ & ${ }^{1} \mathrm{D}$ & -3.002 & -2.985 & -3.125 \\
\hline $3 d^{3}\left({ }^{4} F\right) 4 s$ & ${ }^{5} \mathrm{~F}$ & -3.748 & -3.753 & -3.808 & $3 d^{3}\left({ }^{4} F\right) 4 p$ & ${ }^{3} \mathrm{G}^{0}$ & -3.104 & -3.071 & -3.106 \\
\hline $3 \mathrm{~d}^{3}\left({ }^{4} \mathrm{~F}\right) 4 \mathrm{~s}$ & ${ }^{3} \mathrm{~F}$ & -3.663 & -3.674 & -3.730 & $3 d^{3}\left({ }^{4} F\right) 4 p$ & ${ }^{3} \mathrm{~F}^{0}$ & -3.071 & -3.042 & -3.081 \\
\hline $3 \mathrm{~d}^{3}\left({ }^{4} \mathrm{P}\right) 4 \mathrm{~s}$ & ${ }^{5} \mathrm{P}$ & -3.575 & -3.559 & -3.647 & $3 \mathrm{~d}^{3}\left({ }^{4} \mathrm{P}\right) 4 \mathrm{p}$ & ${ }^{5} \mathrm{P}^{0}$ & -3.001 & -2.974 & -3.017 \\
\hline $3 d^{3}\left({ }^{2} G\right) 4 s$ & ${ }^{3} \mathrm{G}$ & -3.543 & -3.535 & -3.611 & $3 \mathrm{~d}^{3}\left({ }^{4} \mathrm{P}\right) 4 \mathrm{p}$ & ${ }^{5} \mathrm{D}^{0}$ & -2.975 & -2.948 & -2.993 \\
\hline
\end{tabular}
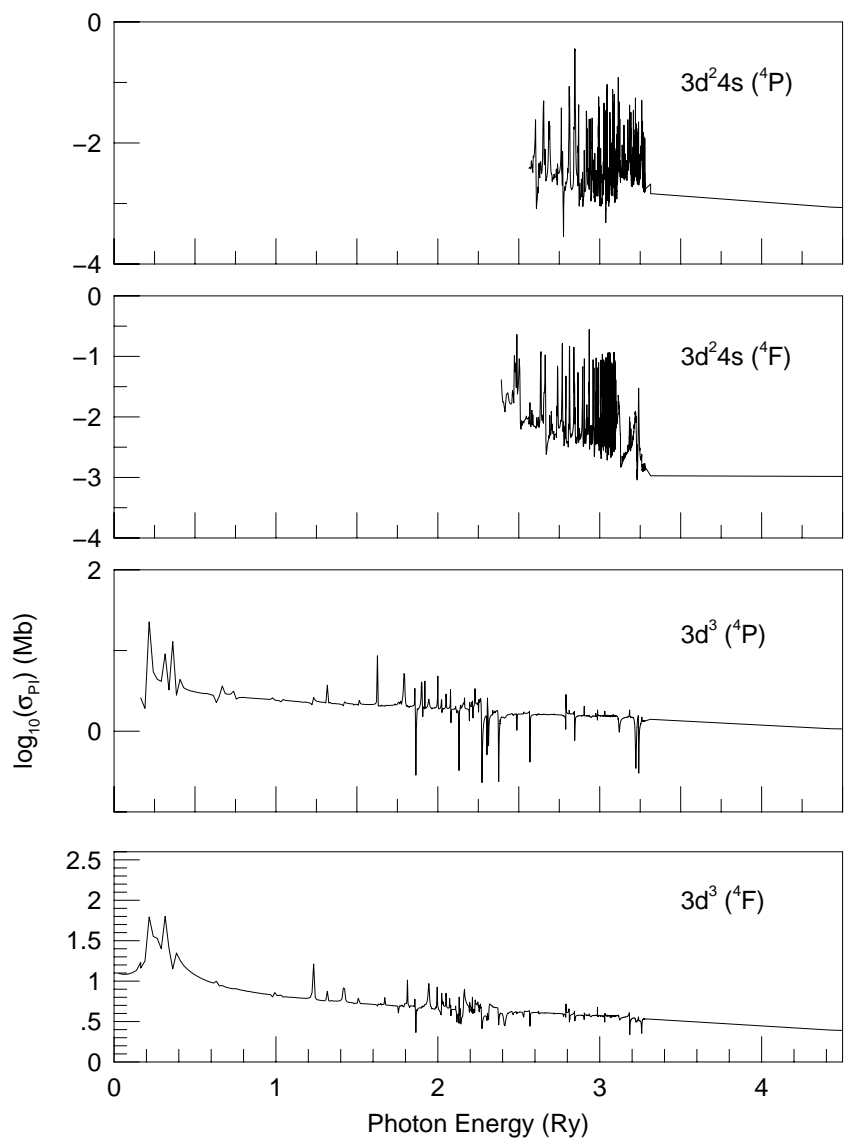

Fig. 3. Partial photoionization cross sections into the ground state and the excited states of Fe VI
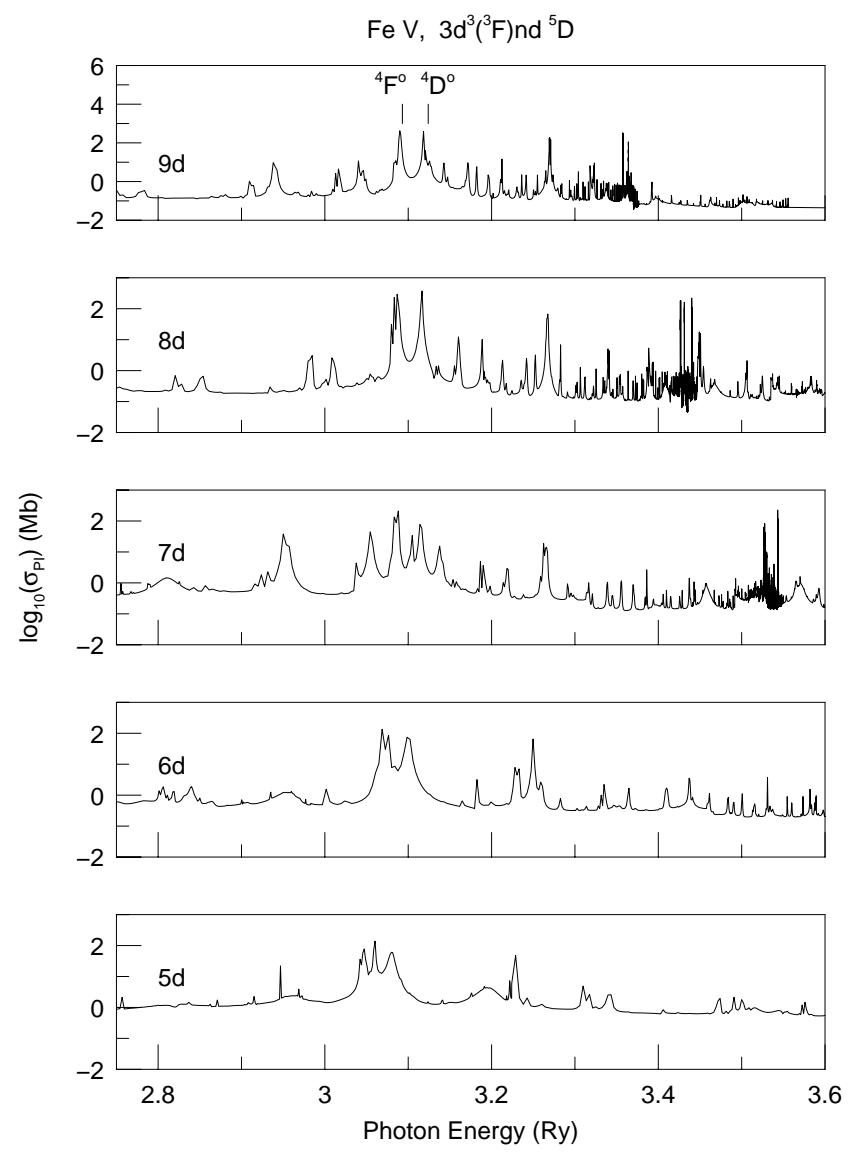

Fig. 4. Photoionization of $\mathrm{Fe} \mathrm{V}$ bound states in a Rydberg series showing the PEC resonances features 
especially for $g f$ values greater than unity. We note that for $g f \geq 0.1$ the overall dispersion is about $16 \%$ which, added to the good agreement between the calculated energies and those observed experimentally, suggests that the data for most such transitions should be accurate to about $10-20 \%$.

\subsection{Photoionization cross sections}

Photoionization cross sections were calculated for all 1818 bound states of $\mathrm{Fe} \mathrm{V}$, obtained in the present calculations, including detailed autoionization resonances resulting from the coupling to states dominated by $3 \mathrm{~d}^{3}, 3 \mathrm{~d}^{2} 4 \mathrm{~s}$, and $3 \mathrm{~d}^{2} 4 \mathrm{p}$ configurations in Fe VI. Figure 2 a shows the photoionization cross section of the $3 \mathrm{~d}^{4}(5 \mathrm{D})$ ground state of $\mathrm{Fe} \mathrm{V}$. In the same figure we have plotted the results of Reilman \& Manson (1979) in the central field approximation, and those of Verner et al. (1993) in the Dirac-HartreeSlater approximation. Both of these approximations neglect the complex correlation effects, such as the interchannel coupling to inner shells, which result in cross sections in the near threshold region with erroneously small values. For instance, it was recently found that the central field approximation underestimates the ground state cross section of Fe I, up to about 1 Ry above ionization threshold, by more than three orders of magnitude (Bautista \& Pradhan 1995). The two earlier results (Reilman \& Manson 1979; Verner et al. 1993) underestimate the photoionization cross section of the ground state of $\mathrm{Fe} \mathrm{V}$, for energies below about $10 \mathrm{Ry}$, by up to a factor of four. Beyond 10 Ry the present cross sections converge well toward the results by Reilman and Manson as might be expected since the electron correlation effects get weaker with increasing energy. However, the calculations by Verner et al. still underestimate the cross section by almost a factor of two. The low values of the photoionization cross section from Verner et al. with respect to those by Reilman and Manson are not understood since both of these calculations are based on a similar approximation. It might be emphasized that the differences between the present $\mathrm{CC}$ calculations and the previous works stems primarily from the inclusion of full coupling to the dominant states of the core ion, particularly those including the inner $3 \mathrm{~d}$ shell, that manifest themselves well below the onset of the 3d-shell ionization through autoionizing resonances and resulting enhancement of the effective background cross section.

In Fig. $2 \mathrm{~b}$ we show the earlier $15 \mathrm{CC}$ results (obtained from TOPbase; Cunto et al. 1993), which also underestimate the cross section for the ground state of $\mathrm{Fe} \mathrm{V}$ by nearly a factor of two in the energy range of interest and neglect much of the resonance structures in the nearthreshold region. This difference arises from the much smaller number of target states in the previous OP calculations for $\mathrm{Fe} \mathrm{V}$.

In practical applications regarding non-LTE spectral models it is often important to determine accurately the level populations in the excited levels of the residual ion following photoionization. This requires that not only should the total but also the partial photoionization cross sections into the excited states of the ionized ion be calculated. Therefore, it is of interest to obtain the partial cross sections for photoionization of the ground state of $\mathrm{Fe} \mathrm{V}$ into at least the lowest few (particularly the metastable) terms of Fe VI. Figure 3 presents these partial cross sections for photoionization of the ground state of $\mathrm{Fe} \mathrm{V}$ into the lowest coupled terms in Fe VI. i.e.

$$
\begin{gathered}
h \nu+\mathrm{FeV}\left[3 \mathrm{~d}^{4}\left({ }^{5} \mathrm{D}\right)\right] \longrightarrow \\
e+\mathrm{FeVI}\left[3 \mathrm{~d}^{3}\left({ }^{4} \mathrm{~F}\right),\left({ }^{4} \mathrm{P}\right), 3 \mathrm{~d}^{2} 4 \mathrm{~s}\left({ }^{4} \mathrm{~F}\right),\left({ }^{4} \mathrm{P}\right)\right] .
\end{gathered}
$$

One interesting feature observed in the photoionization cross sections are the so called photoexcitation-ofcore (PEC) resonances that result from strong dipole couplings between opposite parity terms within the target ion (Yu \& Seaton 1987). Such PEC resonances are seen in Fig. 4 which displays the photoionization cross sections of $\mathrm{Fe} \mathrm{V}$ bound states in the Rydberg series $3 \mathrm{~d}^{3}\left({ }^{4} \mathrm{~F}\right) n d\left({ }^{5} \mathrm{D}\right)$ with $n=5-9$. At the Fe VI target thresholds $3 \mathrm{~d}^{3} 4 \mathrm{p}\left(\mathrm{z}^{4} \mathrm{~F}^{0}, \mathrm{z}^{4} \mathrm{D}^{0}, \mathrm{z}^{4} \mathrm{~S}^{0}\right)$, the incident photon energies equal the energies of the strong dipole transitions from the ground state $3 \mathrm{~d}^{3}\left(\mathrm{a}^{4} \mathrm{~F}\right)$, and large PEC autoionizing resonances are formed, enhancing the effective cross section up to several orders of magnitude above the background. The prominent peaks shown in Fig. 4 correspond to these dipole transition energies $\mathrm{a}^{4} \mathrm{~F} \rightarrow \mathrm{z}^{4} \mathrm{~F}^{0}, \mathrm{z}^{4} \mathrm{D}^{0}$.

\section{Massively parallel R-matrix computations}

The present calculations were carried out using a newly adapted version of the R-matrix codes on the MPP Cray T3D. The Hamiltonian matrixes were computed with parallelized versions of the inner region R-matrix codes (STG1, STG2, and STGH) as described in a previous paper (Bautista \& Pradhan 1995a). The computation of dipole elements with the STG2 code was parallelized according to pairs of coupled total $S L \pi$ symmetries. The calculations of energies in STGB and continuum wavefunctions in STGF were parallelized according to the total $S L \pi$ 's, while dipole $f$-values were calculated by parallelizing over pairs of symmetries in STGBB. Finally, for the computation of photoionization cross sections, the code STGBF was parallelized according to photo-electron energies.

The speed-up of the codes scales linearly with the number of processors up to 32 processors (subject to proper load balancing between the processors), and reduces the computation time by up to a factor of ten or more over serial processing on the Cray Y-MP. 


\section{Conclusion}

Extensive radiative calculations for $\mathrm{Fe} \mathrm{V}$ are presented which are expected to be applicable to a variety of astrophysical problems such as the calculation of improved Iron opacities, non-LTE models, and spectroscopic interpretation of Fe V spectra. The photoionization cross sections are compared with previous theoretical calculations, which are found to be smaller than present results by factors of two to four.

Oscillator strengths for 129.904 dipole allowed transitions among bound terms in $\mathrm{Fe} \mathrm{V}$ were calculated. This data set expands the data set of about 54.000 transitions currently in TOPbase, and is expected to be of significantly improved accuracy. The present data should also be of use in a variety of astrophysical applications which are currently limited by the absence of accurate experimental or theoretical $f$-values. Further work is currently under way to calculate fine structure transition probabilities for both allowed and forbidden transitions, as well as collision strengths for Fe V (Bautista et al. 1995). These results will be published in the IRON Project series.

All radiative data reported here is expected to be included in TOPbase at CDS (Cunto et al. 1993). These data can also be accessed electronically via Internet by request to the author.

The present computations were carried out on the MPP Cray T3D, which results in a significant speedup of such large scale ab-initio calculations.

Acknowledgements. I would like to thank Prof. Anil K. Pradhan for suggesting this work and for discussions and comments. This work was supported in part by the U.S. National Science Foundation (PHY-9421898) grant for the Iron Project. The computations were carried out on the Cray T3D at the Ohio Supercomputer Center (OSC).

\section{References}

Bautista M.A., 1996, A\&AS (submitted)

Bautista M.A., Pradhan A.K., 1996, A\&AS 115, 551

Bautista M.A., Pradhan A.K., 1995, J. Phys. B: At. Mol. Phys. 28, L173

Bautista M.A., Nahar S.N., Peng J.F., Pradhan A.K., Zhang H.L., 1995, in Astrophysics in the Extreme Ultraviolet. In: Bowyer S. (ed.), IAU Symposium No. 152

Berrington K.A., Burke P.G., Butler K., et al., 1987, J. Phys. B: At. Mol. Phys. 20, 6379

Cunto W., Mendoza C., Ochsenbein F., Zeippen C.J., 1993, A\&A 275, L5-L8

Eissner W., Jones M., Nussbaumer H., 1974, Comput. Phys. Commun. 8, 270

Eissner W., 1991, J. Phys. IV (Paris) C1, 3

Hummer D.G., Berrington K.A., Eissner W., et al., 1993, A\&A 279, 298

Nahar S.N., 1995a, A\&AS 293, 967

Nahar S.N., 1996, Phys. Rev. A 53, 1545

Nahar S.N., Pradhan A.K., 1994, J. Phys. B: At. Mol. Phys. 27,429

Pradhan A.K., 1995, in Astrophysics in the Extrema Ultraviolet. In: Bowyer S. (ed.), IAU Symposium No. 152

Reilman R.F., Manson S.T., 1979, ApJS 40, 815

Seaton M.J., Yu Yan, Mihalas D., Pradhan A.K., 1994, MNRAS 266, 805

Sugar J., Corliss C., 1985, J. Phys. Chem. Ref. Data 14 Suppl. No. 2

Vennes S., 1995, in Astrophysics in the Extrema Ultraviolet. In: Bowyer S. (ed.), IAU Symposium No. 152

Verner D.A., Yakovlev D.G., Band I.M., Trzhaskovskaya M.B., 1993, At. Data. Nucl. Data Tables 55, 233

Yu Yan, Seaton M.J., 1987, J. Phys. B: At. Mol. Phys. 20, 6409 